from the Royal Observatory, Greenwich, to have begun at 0928 G.M.T. was, we suggest, the cause of this burst of ionization, which increased the normal amount in this region by more than 100 per cent. The photograph shows a second reflection from this height of $125 \mathrm{~km}$., indicating that no marked absorption in or below the normal $E$ region was produced during the eruption. These observations indicate that the quality of the ionosphere for the propagation of radio waves is sometimes improved as the result of a bright solar eruption.

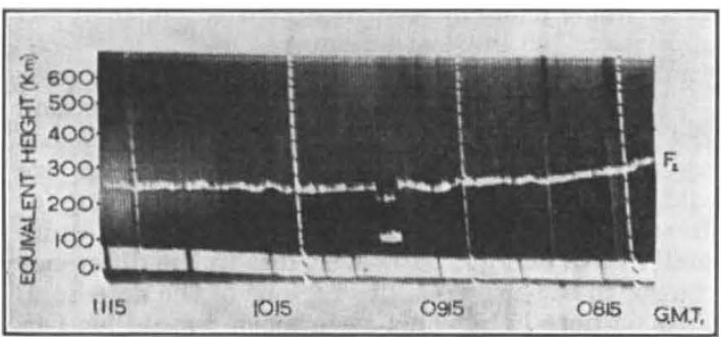

IONOSPHERIC DISTURBANCE BEGINNING AT 0927, G.M.T. on October $1,1937$.

It is now well known ${ }^{1}$ that there have been an increasing number of occasions during the last few years when, during a bright solar eruption, radio signals have disappeared completely. At such times, the condition of the ionosphere is similar to that described in $1933^{2}$ as the 'no-echo' condition, and is due to the production of ionization at abnormally low levels, the consequent absorption thereby masking the normal reflecting regions.

We deduce therefore that on a majority of occasions on which bright solar eruptions take place, ionization is produced in the ionosphere, and that it depends primarily upon the height at which it is produced whether radio transmission conditions are better or worse. It follows therefore that the initial comparisons of the worst radio transmission conditions and bright solar eruptions must be extended to include this more recent observation before the effects of a bright solar eruption on the ionosphere can be completely assessed.

The work described above was carried out as part of the programme of the Radio Research Board and this note is published by permission of the Depart ment of Scientific and Industrial Research.

R. NAISMITH.

W. J. G. Beynon.

Radio Department,

National Physical Laboratory,

Teddington, Middlesex. July 7 .

${ }^{1}$ For bibliography, see Newton and Barton, Mon. Not. Roy. Ast. Soc., 594 (June 1937).

'Appleton, Naismith and Builder, NATURE, 132, 341 (1933).

\section{Crystal Structure Models}

TABLE tennis balls have often been used in making models of crystal structures, and the purpose of this letter is to direct attention to two ways of using them which, so far as we are aware, are new.

(1) Models of crystal structures of inorganic substances are clearest when the atomic centres are indicated by balls and these are joined by rods. The following method may be used to connect table tennis balls by rods of celluloid, a convenient diameter for which has been found to be $3 / 16$ in. The ends of the rod are dipped in acetone for about a minute when they swell and become gelatinous on the surface. Meanwhile, the surfaces of the balls to be joined are rendered slightly gelatinous by moistening them with acetone. The rod is now placed in position between the balls and, if necessary, held there for about 20 sec. The rod and balls now adhere to one another sufficiently strongly for it to be possible to continue building the model. 'Durofix' may be used as the cement, but the join is not so strong as that obtained with acetone. Celluloid rods can be easily cut to any required length and this greatly facilitates the making of models with correct interatomic distances.

(2) In studying the structures of organic substances, it is often necessary to see how molecules pack into the unit cell. The distance between neighbouring carbon atoms in a molecule is about $1.5 \mathrm{~A}$., while the distance between carbon atoms in neighbouring molecules is not usually less than $3 \cdot 4 \mathrm{~A}$. A model representing the space occupied by any one molecule can therefore be made by cutting off spherical caps from the table tennis balls and fitting the balls together so that the distance between their centres is $\frac{2}{3}$ in. Such model molecules can then be packed into the unit cell with their surfaces touching. To cut off the spherical caps the balls are held in a split brass tube of internal diameter slightly less than $1 \frac{1}{2}$ in., mounted on a lathe, and a razor blade is used as a cutting tool.

The accompanying photographs show similarly orientated models of naphthalene, $\mathrm{C}_{10} \mathrm{H}_{14}$, constructed according to both methods. The model with rods

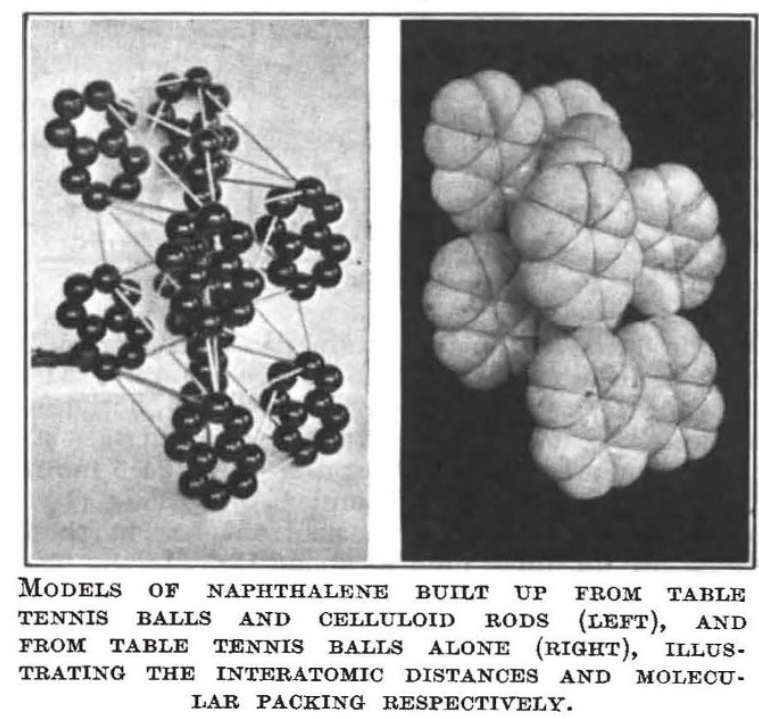

is valuable in teaching, and the packing model is more useful in research work when it is required to discover the geometrically possible molecular orientations.

W. A. Wooster.

Department of Mineralogy and Petrology,

Cambridge.

Crystallographic Laboratory,

G. Котт. Cambridge. 